\title{
A Remote Educational Video Dynamic Image Sharp Processing Algorithm
}

\author{
Tang Qianjun, Zhang Yan \\ Physical and Electronic Engineering School, Leshan Normal College \\ Leshan, Si Chuan, China 614000
}

Keywords : Distance education, Network fault, Neural network

\begin{abstract}
In distance education network transmission process, because transmission distance is too long, transmission network will be affected by complicated external environment factors, which leads to network failure and failure in remote education video image formation, and finally causes unsmooth transmission. This paper puts forward a distributed network fault detection technology to perform fault detection for remote education transmission network nodes and characteristic analysis of the use of network fault by using genetic neural network, accurately locate fault node area so as to realize the remote education network's fault detection. Experiments show that this method can avoid distance education network fault resulted from long transmission distance and improve the transmission efficiency of remote education video image.
\end{abstract}

\section{Introduction}

With the highlight of imbalance problem of the rapid development of education cause and different areas' education resources, it has become the focus of education research that the use of remote education can improve the teaching quality of teaching resources. [1]. The wide application of Internet and other communications facilities makes education tend to globalization which borns a lot of innovation and reform opportunities. At present, education can not only be implemented through the class and still can be provided through various communication media and communication network. This will cause the transformation from traditional classroom teaching process to the virtual classroom teaching. But the ultimate goal is to make education open: the enlargement of teaching time and space, more wide teaching object, more open education concepts, and more free teaching practice. At present, the teaching resources of developing areas in China are abundant with relatively strong faculty which results a big gap of the education resources between developed regions and remote areas leading to relative poor education resources of remote areas [2]. The use of remote education can improve education resource allocation imbalance problem and the level of education in remote areas [3]. Distance education resources need long distance transmission which will cause a lot of noise defect in the process of distance education video image data transmission due to the complicated environmental interference [ $4 \sim 5]$. Therefore, This paper puts forward a distributed network fault detection technology to perform fault detection for remote education transmission network nodes and characteristic analysis of the use of network fault by using genetic neural network, accurately locate fault node area so as to realize the remote education network's fault detection. Experiments show that this method can avoid distance education network fault resulted from long transmission distance and improve the transmission efficiency of remote education video image.

\section{Network Fault Characteristics Analysis}

Define 1: network state $\mathrm{S}$ is a binary group $S(U, E) ; U$ is a non-empty limited objects set which is called tube object space and noted as $U=\left\{m_{i}, i=1,2, \cdots\right\} ; m$ is the network object; $E$ is the respective state of network entity, $E=\left(e_{1} f_{1}, e_{2} f_{2}, \cdots e_{n} f_{n}\right)$ and $e_{i}$ is state property; $f_{i}$ is the 
corresponding attribute parameter. Network status information refers to the behavior information extracted from the state of the network entity $E$ in network operation.

Definition 2: network entity can be defined as a $\operatorname{triad}(A, E, G)$, in which $G: A \times E \rightarrow E$ is system migration. $\mathrm{G}$ is the mapping function of state change; $\mathrm{A}$ is an events set caused by physical state change. Network local state can be divided into $\mathrm{L}$ irrelevant features $C=\left\{c_{1}, c_{2}, \cdots, c_{L}\right\}$; $\mathrm{L}$ is decided by the domain node nature. Network fault is a state change from normal state $c$ to the abnormal state $c^{\prime}$.

Network status information is gained by information collection device. Data collection device roll polls the relevant management information bank variables based on simple network management protocol and get the current network running state E. Data collection device will transpose $\mathrm{E}$ transformation and convert it into complex state information:

$N=\left(e_{1} f_{1}, e_{2} f_{2}, \cdots e_{n} f_{n}\right)^{T}$ (1) set a threshold value $T_{1}$ for entity state attribute parameter $f_{i}(i=1,2, \cdots, n)$. According to entity performance characteristics, $T_{1}$ can be got by expert knowledge or repeated experiment adjustment and establish a comparison function:

$$
\delta\left(f_{i}\right)=\left\{\begin{array}{l}
0, f_{i}<T_{1} \\
1, f_{i}=T_{1},(i=1,2, \cdots, n) \\
2, f_{i}>T_{1}
\end{array}\right.
$$

Substituted $f_{i}$ for $\delta\left(f_{i}\right)$, after pretreatment, the state information is $N^{\prime}=\left(e_{1} \delta\left(f_{1}\right), e_{2} \delta\left(f_{2}\right), \cdots, e_{n} \delta\left(f_{n}\right)\right)$. Let $x_{i}=e_{i} \delta\left(f_{i}\right), i=1,2, \cdots, n$ :

$$
M=\left(x_{1}, x_{2}, \cdots, x_{n}\right)^{T}
$$

Therefore, $\mathrm{n}$ dimension vector is used to express the local network characteristics.

\section{Distance Education Network'S Fault Diagnosis Based on Genetic Neural Network}

\section{Genetic Algorithm}

Genetic algorithm adopts natural evolution model such as selection, crossover and mutation, etc. At the beginning, the initial population (randomly generate $\mathrm{N}$ individuals), calculates each individual's fitness function. In this process, select individuals in accordance with the fitness. Father generation requires gene recombination. All progeny vary according to certain probability, and then progeny fitness is recounted. Progeny of higher fitness are inserted into the population and replace the father generation of lower fitness. So a new generation is formed. Repeat this process until meet optimal criterion.

Genetic algorithm is mainly realized through crossover, mutation and selection arithmetic. Generate next generation chromosome by crossing and mutation operation, which is called generations. According to the size of the fitness, select a number of individuals from previous generations and latter generations. As the next generation group, then continue to evolve. So after several generations, algorithm converges in the best chromosomes. It might just be the optimal solution or suboptimal solution. Genetic algorithm is to finish encoding solutions first. This paper's filter designer is a nonlinear function optimization problem, so this paper uses real number's coding method. First express the chromosome as vector form [9] :

$X=\{X(0), X(1), \cdots, X(N-1)\}$

In it, $X(i) \in[0,1], \quad i=0,1, \cdots, N-1, X(k) \in[0,1], k=0,1, \cdots, N-1 / 2$ can be got through the following mapping relation:

$X(k)=[h(k)+1] / 2$

In which, $h(k) \in[-1,1], k=0,1, \cdots, N-1 / 2$.

Next, suitable fitness function need to be chosen. The main goal of FPGA digital filter's optimization design is to get the minimum of the upper and lower formulas. This paper adopts the fitness function: 


\section{$f=1 / E^{2}$}

\section{Crossover and Mutation Operator}

In choosing a crossover operator, this paper adopts probability $p_{c}$ to randomly segment two elder individuals, then regroup to get two new individuals. Then based on the number of division points, each parent individual randomly chooses $m$ non-repeat intersection points, so interlace operation is realized.

Variation is to arrange and mutate genes on a certain position of individual chromosome based on probability $p_{m}$. The father individual vector is expressed as

$x=\left(x_{1}, x_{2}, \cdots, x_{k}\right)$

In it, the component $x_{i}$ is selected as a variation based on probability $p_{m}$.

\section{Genetic Algorithm Optimizes Neural Network Weights}

BP network is studied by using genetic algorithm, and the steps are as follows:

(1) Initialize population $\mathrm{P}$, including cross scale, crossover probability $p_{c}$ and mutation probability $p_{m}$. This paper adopts real number for coding. The initial population's value is 30 ;

(2) Calculate each individual's evaluation function, at the same time sequence it and select network individual based on the probability value of the following formula:

$$
p_{s}=f_{i} / \sum_{i=1}^{N} f_{i}
$$

In it, $f_{i}$ is fitted value of individual $i$, which can be used to measure the error sum of squares and E, namely:

$$
f(i)=1 / E(i) \quad E(i)=\sum_{p} \sum_{k}\left(V_{k}-T_{k}\right)^{2}
$$

In it, $i=1,2, \cdots, N$ is the chromosome number; $k=1,2, \cdots, 4$ is output layer node number; $p=1,2, \cdots, 5$ is learning sample numbers.

(3) Probability $p_{c}$ is used to crossover operate the individuals $G_{i}$ and $G_{i+1}$ and generate new individuals $G_{i}^{\prime}$ and $G_{i+1}^{\prime}$. Directly copy individual without crossover operation;

(4) Probability $p_{m}$ mutates to produce $G_{i}$ new individual ${ }_{j}^{\prime}$;

(5) Insert the new individual into population $p$, and calculate the new individual's evaluation function;

(6) If satisfactory individual is found, then end, or turn to(3)

After reaching the required performance indicators, decode the optimal individual in eventually group to get network connection weight coefficient after optimization. Construct the corresponding adaptive fuzzy Petri nets and train fault phenomenon weights in rules, which can realize the fault diagnosis, and the specific steps are shown as follows:

(1) Establish accessible set $R S(P)$ of each library $P$, namely get set IRS (P) and the adjacent library collection AP $(\mathrm{P})$.

(2) If sign library is $P_{0}$, to any library, if $P_{0} \in I R S\left(P_{k}\right)$, compare all rules' confidence corresponding $P_{k}$ and $P_{0}$, select the database $P_{i}$ corresponding maximum confidence degree rules, and give access mark $v_{i}=1$.

(3) To $P_{i}$, find including libraries in $\operatorname{IRS}\left(P_{i}\right) \operatorname{IRS} \operatorname{IRS}\left(P_{k}\right)$, compare confidences corresponding the rules, select the database corresponding the maximum confidence degree rules, and then move to (4), until we find fault library, and set it $P_{i}$.

(4) If $P_{i}$ has the corresponding premise database in $\operatorname{IRS}\left(P_{i}\right)$, then turn to (3) continue to search. If not, turn to (5) to process. 
(5) If $P_{i}$ has the adjacent library $\operatorname{AP}\left(P_{i}\right)$ in $\mathrm{AP}$, the system inquires the severity based on the corresponding proposition of $P_{i}$ and $A P\left(P_{i}\right)$. Calculate input total confidence = lose the confidence of $\times$ corresponding weights + adjoining library of confidence + corresponding weights according to the system preset fuzzy degree quantitative table or experts' direct input corresponding $\alpha$. In the reasoning process, search all the path which can cause the sign in the knowledge base. According the size of change credibility and depth priority principle, select successively the path. If the input library of a change matches the fault fact, then start the transition; Otherwise always repeat the process until find fault database or without finding the fault database.

\section{Simulation Results}

In order to collect the network equipment's MIB value of all states, we use the existing equipments of the laboratory to set up a experiment network simulating distance education network, use two Cisco 2600 routers to connect two local area networks. Each LAN has six computers. Switchboard is used to connect routers. Use a network management station to connect the router's console port. Control the router and collect MIB variable data. Network's topology results are shown in Fig 1.

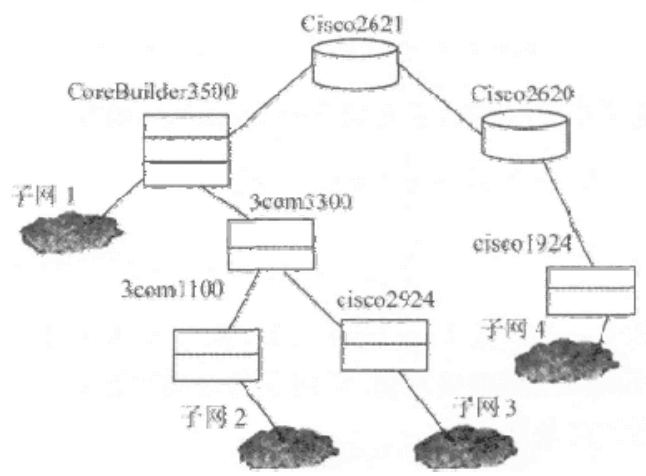

Fig.1 Topology of the network

\section{Simulation Network Fault Phenomenon}

In order to collect the MIB variable data under network fault state, we simulated some network faults in network system. The specific steps are as follows: modify the configuration of network equipments and make it unable to work properly, so that some fault types expected in network system appear; Artificially set device interface as managerial closed state or mismatch of interface rate between equipments, etc., by using some network application softwares to produce a large number of packets, and make the network resource depletion in order to produce network fault; Forcefully pull and plug network interface connection lines to result in sudden interruption of network communication failure.

\section{Network Fault Diagnosis Of This Paper Algorithm}

Divid the data in table 1 into two parts. The first 15 data sets is the training sample set, and the latter 5 data groups are test samples. Genetic neural algorithm is used to get optimal parameters. The optimal parameters model is used to test the effectiveness of test set to illustrate the effectiveness of the algorithm. To illustrate the effectiveness of the algorithm, compare BP neural network model (BPNN) and this paper's model. Diagnosis results are in Tab 1.

Tab.1 Diagnosis comparison

\begin{tabular}{|c|c|c|c|c|c|c|c|c|c|c|c|c|}
\hline \multirow{2}{*}{ Sample } & \multicolumn{4}{|c|}{ raw data } & \multicolumn{4}{|c|}{ this paper's algorithm } & \multicolumn{4}{|c|}{ BPNN } \\
\hline & $B_{1}$ & $B_{2}$ & $B_{3}$ & $B_{4}$ & $B_{1}$ & $B_{2}$ & $B_{3}$ & $B_{4}$ & $B_{1}$ & $B_{2}$ & $\boldsymbol{B}_{3}$ & $B_{4}$ \\
\hline 16 & 1 & 0 & 0 & 0 & 1.01 & 0 & 0 & 0 & 0.98 & 0.1 & 0.02 & 0 \\
\hline 17 & 0 & 1 & 0 & 0 & 0.01 & 1 & 0.02 & 0 & 0.3 & 0.98 & 0.04 & 0.1 \\
\hline 18 & 0 & 0 & 0 & 1 & 0.03 & 0 & 1.02 & 0.01 & 0.6 & 0.2 & 0.3 & 0.86 \\
\hline 19 & 1 & 0 & 0 & 0 & 0.99 & 0 & 0 & 0.04 & 1.2 & 0.03 & 0.1 & 0.02 \\
\hline 20 & 0 & 1 & 0 & 0 & 0 & 1.02 & 0 & 0.04 & 0.2 & 0.87 & 0 & 0.12 \\
\hline
\end{tabular}


From Tab 1, it is known that the network fault diagnosis results are the most close with the actual fault, whose diagnosis accuracy reaches $100 \%$. Although the output results aren't the same with original data, the differences are very small. This verifies the method presented in this paper has strong ability of fault diagnosis. In the diagnosis of sample 18 with BPNN, the difference between the output and the original data is large, in it, B1 and B4 are greater than 0.5 , so that the network manager is difficult to judge the specific type of the fault. From the diagnosis results of this method and BPNN, it can be seen that in view of the small samples' network fault diagnosis, this method's diagnostic accuracy is obvious than BPNN.

\section{Concluding}

This paper puts forward a distributed network fault detection technology to perform fault detection for remote education transmission network nodes and characteristic analysis of the use of network fault by using genetic neural network, accurately locate fault node area so as to realize the remote education network's fault detection. Experiments show that this method can avoid distance education network fault resulted from long transmission distance and improve the transmission efficiency of remote education video image.

\section{References}

[1] Eftekhari A., R. R Lewis. Feasibility ofFixed Wireless Access[J]. Proc. Of ISART2003,Colorado USA,Mar.2003:111-118.

[2] M.Z.Win, and R.A.Scholtz,Ultra-wide bandwidth time-hopping spread-spectrumImpulse radio for wireless multiple access

[3] communications[J].IEEE Transactions onCommunications, 2000,48, (4)679-691.

[4] Lee Kyung Chang, Lee Suk1. emote Controller Design of Networked Control System Using Genetic Algorithm[J].ISIE2001.Pusan.KOREA inIEEE12001:1845-1850

[5] Carlo Dell'AQUILA,Francesco DI TRIA, Ezio LEFONS, and Filippo TANGORRA.An Academic Data Warahouse[J].Proceedings of the 7th WSEAS International Conference on Applied Informatics and Communications,Athens,Greece, August 24-26,2007:229-235.

[6] PAlkis Simitsis, Panos Vassiliadis, PTimos Sellis. Optimizing ETL Processing in Data Warehouses[J]. IEEE Computer Society.2005:564-575. 